\begin{tabular}{lcr}
\hline \multicolumn{3}{c}{ ANNALES } \\
UNIVERSITATIS MARIAE CURIE-SKŁODOWSKA & \\
& LUBLIN - POLONIA & \\
VOL. LXXIII, 1 & SECTIO AA & 2018 \\
\hline
\end{tabular}

\title{
Artificial skin composites
}

\author{
Agata Ładniak \\ Department of Interfacial Phenomena, Faculty of Chemistry, \\ Maria Curie-Skłodowska University in Lublin; \\ e-mail: agata.gozdecka@umcs.pl
}

Skin injuries are a health problem and can lead to serious, significant deterioration in the quality of life and, consequently, even illness and disability. Therefore, after wounding, immediate regeneration of the tissue is necessary to avoid further complications and pathogenesis. Consequently, many wound healing strategies have been developed, leading to the progress in constructing of multifunctional tissue substitutes for the skin, biomembranes, scaffolds and intelligent dressings. The field of science focusing on the creation of the abovementioned products is tissue engineering (TE). Its main goal is to find a system that is able to replace or be a model that perfectly mimics the form and function of the skin. Research carried out on such constructs is mainly based on the analysis of mechanical properties (porosity, elasticity), as well as the assessment of the impact of individual components on processes related to the formation of new tissue as cell proliferation and differentiation, proliferation, angiogenesis through in vivo studies (using animal models: mice, New Zealand rabbits) and in vitro (most often using mouse fibroblasts - L929). Skin constructions may have potential applications as wound dressings or skin substitutes in cases of severe skin damage.

Keywords: titanium dioxide, chitosan, hyaluronic acid, skin substitutes 


\section{INTRODUCTION}

The skin is the largest organ of the human body and consists of three layers: the epidermis, dermis and subcutaneous tissue. It constitutes the first physical barrier protecting against mechanical, chemical, osmotic and thermal damages. In addition, it prevents the penetration of microorganisms and regulates body temperature. Skin that has permanent direct contact with the external environment may be damaged.

A scientific discipline which, by means of alternative solutions, significantly changes the approach to treatment by means of transplants and replenishes of organ or tissue defects is tissue engineering (TE), thus becoming the driving force behind the development of regenerative medicine.

Biomedical engineering is an interdisciplinary field of knowledge, which consists of technical, biological and medical sciences. Because the area of biomedical engineering is very broad, continuous cooperation of specialists is necessary. However, success in tissue engineering would not be possible without the development of new biomaterials that significantly accelerate the regeneration of damaged tissues. In 1987, during the European Society of Biomaterials Conference, a group of experts defined biomaterials as abiotic materials used as medical devices that are intended to interact with biological systems. In the present medical terminology, the biomaterial is defined as any substance other than the drug, or a combination of natural and/or synthetic substances which task is to supplement or replace tissues, organs or parts thereof or fulfill their function [1]. The role of cell scaffolds, in addition to maintaining cells in space, is to ensure conditions as similar as possible to those that occur naturally in the body.

Most organs have a characteristic three-dimensional structure that requires a special solid support to be formed from surrounding cells. The frame constituting such a substrate is called scaffolding, matrix or artificial extracellular matrix. It performs the same function as a natural matrix, that is, it participates in such processes as: proliferation, differentiation and cell biosynthesis. In addition, it prevents inflow of cells from neighbouring tissues and substances produced by fibroblasts, which massively colonize the cavity - this is very important because their presence hinders the regeneration of the organ. Cell-matrix interaction is the main factor affecting cell 
binding. Biomaterials are not only a support but also active promoters of tissue regeneration. The dream of any tissue engineer is to create a biomaterial that would have similar mechanical properties to those that characterize real tissue, and that would provide a surface allowing the adherence of the cell and the occurrence of appropriate biological processes. In addition, it is important that its show biocompatibility. So, one that integrates with the surrounding tissue, supports regeneration, and its degradation products do not cause toxic effects.

A good biomaterial which would ideally imitated the structure of the extracellular matrix (ECM) must have appropriate physical and chemical properties. First of all, it should have a proper texture, because the shape of the implant affects the physiological reaction of the body. It is expressed through porosity - a parameter that determines the amount of free spaces (micropores) that will be settled by the cells. At the same time, cells should be free to migrate, multiply and have easy access to nutrients and the ability to discharge side-products of metabolism, which requires that the micropores inside it are well connected with each other. The size and number of pores has a huge impact on the penetration of cells into the implant, which consequently has a positive effect on the regeneration of damaged tissue. Macropores increase the cell attachment to the surface, and are essential for cell growth and proliferation in porous scaffolds. Mesopores, on the other hand, allow efficient delivery of nutrients to cells [2]. Against, the matrix structure can not also be too "spongy" and must maintain excellent mechanical strength. The second critical issue for tissue regeneration is the absorption kinetics of the material.

Materials that have the potential to be used as skin substitutes should be created by substances of natural origin. These are various types of proteins, peptides (collagen, fibrin), polysaccharides, but also synthetic ones, such as ceramics, metals and their composites. Particularly noteworthy is the silk [3] produced by insects, which is known as a protein fiber with very attractive features for tissue engineering applications such as: significant mechanical strength and extended shelf life, the ability to increase: adhesion, growth and differentiation of the cells, such as fibroblasts and keratinocytes. In addition to peptide matrices, other polymers that create artificial, but at the same time quite "friendly" ECMs are polysaccharides. They are quite often used because of the extraordinary easiness of processing, 
as well as their consistency, which can easily perform to a gel state, which facilitates delivery to the wound. Among the most commonly used polysaccharides are agarose, alginate, hyaluronan and chitosan. Recently, polymer-based materials have gained unusual interest due to their biodegradability in a short time, which can also be adapted according to necessity. Their special advantage is also the biocompatibility, which is extremely important because the materials used as skin/tissue substitutes remain in direct contact with them for a long time. This made the polymers have a lot of attention in biomedical applications and can be functional skin substitutes [4].

In addition to natural substances for the production of biomaterials, synthetic polymers - polyhydroxy acids, especially polyglycolide (PGA) and polymers of lactic acid - are also often used. Their chief advantage is the ability to obtain repeatability, thanks to a strictly defined chemical composition, which also helps to maintain control over the mechanical properties, shape and degree of degradation of the finished product. However, they often require additional surface modification due to the frequent absence of the stem cell adhesion sites, and the lack of biocompatibility, as a result of which their decomposition products may elicit the immune response.

The most important goal of tissue engineering is to obtain biological material that allows replacement or restoration of basic functions of damaged tissues. The use of tissue engineering allows solving problems related to organ transplants, for example, insufficient donors or the use of immunosuppressive drugs, and does not require the apply of artificial prostheses, which are characterized by low biocompatibility.

The first step in the production of biomaterials with potential use as skin substitutes is to check their properties to accelerate wound healing, and thus affect the speed of proliferation and angiogenesis processes associated with the formation of a new batch of epidermis. Designing scaffolds for the skin in tissue engineering requires adequate comprehensive knowledge of the processes occurring during wound healing, types of wounds, pathological conditions of patients, healing mechanisms and optimal properties of the ideal dressing.

The following brief overview presents recent, most promising literature reports in the field of soft tissue engineering, with a focus on natural substances such as for example: chitosan, hyaluronic acid 
and titanium dioxide. These achievements gain a lot in the face of a more and more real vision of regenerative medicine.

\section{REVIEW OF THE LITERATURE}

\subsection{Combinations of hyaluronic acid and chitosan}

Hydrogels and wound dressings based on chitosan are biocompatible, biodegradable and non-toxic, and can also be used as drug delivery matrix molecules. There are usually used in combination with other biomaterials, due to chitosan low solubility in aqueous solutions, however, its modification is not a major problem due to the availability of functional groups. The cationic nature of this polysaccharide helps in the formation of polyelectrolyte complexes with a wide range of anionic glycosaminoglycans (GAGs) [5, 6]. One of the GAG abundant in native dermis is hyaluronic acid (HA). This polymer strengthens the proliferation of both kind of cell, fibroblasts and keratinocytes, and has been successfully used to reconstruct skin and epidermis.

The use of hydrogels as a regenerative scaffold is a promising approach to the treatment of skin defects by inducing skin reconstruction. In order to develop a good skin substitute, Zhu et al. [7] analyzed hydrogels that mimic the extracellular matrix of the human body and can be used for tissue engineering scaffolds. Hydrogels were prepared by adding hyaluronic acid (HA) and carboxylated chitosan (Ch) to human collagen (HLC is a water-soluble protein whose molecular weight is 97,000 ) using transglutaminase as a cross-linking agent. Systems containing various concentrations of HA and Ch: GEL2 (HLC + 1\% HA), GEL3 (HLC + 2\% Ch) and GEL4 $(\mathrm{HLC}+1 \% \mathrm{HA}+2 \% \mathrm{Ch})$ were prepared using a system without polymers as a reference system - GEL1.

The morphology of freeze-dried hydrogels was determined using SEM. Analysis showed that hydrogels have a pore structure suitable for tissue regeneration applications. As the best were recognized GEL3 and GEL4 showing a pore diameter of 64.58 and $79.06 \mu \mathrm{m}$ and a total porosity of $91.4 \pm 2.01 \%$ and $92.62 \pm 3.24 \%$, respectively. However, kinetic studies have shown that hydrogels exhibit significant swelling properties at comparable rates in both deionized water and saline, which indicates their excellent hydrophilic 
properties. The highest swelling ratio was obtained for GEL4, which was determined by the larger pore size and overall porosity as well as the presence of a significant number of hydrophilic groups derived from HA and Ch. It is very valuable property because hydrogels with different swelling abilities are suitable for various types of wounds and exudate levels.

At a later state research, cytoblasts (L929) were used to assess cytotoxicity and the ability of hydrogels to maintain cell viability and adherence. Hydrogels showed very low cytotoxicity to proliferation of L929 cells after 1 day. However, culturing of cells with hydrogel extracts led to a significant increase in the number of cells after 5 days, where the cell growth rate reached 155.59, 172.90 and $170.76 \%$ for the GEL2, GEL3 and GEL4 extracts respectively. In addition, SEM images indicated that hydrogels support cell adhesion. In addition, good tissue compatibility of the hydrogels with the animal model was demonstrated (new-born rabbits were used), because they healed wounds after 14 days of treatment and over time. The hydrogels gradually degraded in proliferative inflammatory cells, which was consistent with the cycle of skin tissue healing. The total assessment of the tested materials allowed to state that they can be successfully used for skin repair, drug delivery and tissue engineering applications.

In turn, Berce et al. [8] demonstrated that the combination of chitosan, sodium hyaluronate and resveratrol, supports the formation of granulation tissue with reduced neutrophil infiltration in the mouse model. In addition, the systems produced had a spongy structure - suitable for replacing the extracellular matrix, and showed no bacterial contamination compared to the control, and promoted local angiogenesis and re-epithelial formation. In another paper, Croisier et al. [9] noted that the combination of $\mathrm{Ch}$ and $\mathrm{HA}$ also strengthens other important processes in the reconstruction of the epidermis like cell adhesion and proliferation. Nanofibres generated by electrospinning of poly ( $\varepsilon$-caprolactone) coated with the layer-bylayer method of polyelectrolyte polysaccharides ( $\mathrm{Ch}$ and $\mathrm{HA}$ ) promoted the growth of keratinocytes.

\section{2. $\mathrm{TiO}_{2}$ and $\mathrm{Ag}$ nanoparticles enhancing the properties of substitutes}

Sometimes the use of polymers themselves leads to materials with good biocompatible but poor mechanical properties. The 
solution in such cases may be the introduction to nanomaterial systems that have unique mechanical, electrical and optical properties. The use of nanomaterials can contribute to the promotion of more specific interactions stimulated at the cellular level, which ultimately leads to more effective creation of new tissues. Nanoparticles incorporated into bioactive components contribute to the (slow and prolonged) release of the active substance at the target site and also increase the stability of the molecules [10].

Particularly notable are nanoparticles of copper, silver, zinc oxide, titanium dioxide, which can be successfully incorporated into scaffolding, in order to improve their mechanical strength, required for the matrix. In addition, they have a strong antibacterial activity and are characterized by a large surface area and high drug loading capacity $[11,12]$. Besides, it has been reported that the use of a thin layer of $\mathrm{TiO}_{2}$ has definitely improved the adhesion in early human healing of the oral mucosa [13]. However, Khalid et al. [14] noted that the bacterial cellulose composites with $\mathrm{TiO}_{2}$ nanoparticles improved wound healing potential and enhanced tissue regeneration compared to the unmodified polysaccharide. The research was supplemented with antibacterial analysis against Escherichia coli, Salmonella typhimurium, Citrobacter fruendii, Pseudomonas aeruginosa, Staphylococcus aureus and Staphylococcus aureus resistant to methicillin - the most frequently infecting wound. It was found that cellulose and $\mathrm{TiO}_{2}$ nanocomposites caused a significant inhibition of the pathogen, mainly through mechanisms involving the destruction of cell wall integrity, cell membrane, alteration of cell pathways and inhibition of enzymes.

The combination of organic substances such as polysaccharides and inorganic substances can increase the tensile strength of the tissue, as demonstrated by research on nanocomposites such as chitosan-bentonite [15], or chitosan-polyvinylpyrrolidone- $\mathrm{TiO}_{2}$ nanoparticles [16]. In addition, the use of chitosan in this type of material results in an increase in antibacterial activity against Gramnegative bacteria and proliferation of vascular endothelial cells in the mouse model [17].

However Ye et al. [18] have developed composites based on gelatin, chitosan and silver particles, analyzing several systems with different metal content. As a result of the research, they drew a lot of important conclusions: (i) the pore size of the tested materials was about 100-250 $\mu \mathrm{m}$, which promotes liquid absorption, and (ii) they 
showed an increase in pore size with increasing AgNP concentration, (iii) TEM analysis confirmed the presence of $\mathrm{Ag}$ with a particle diameter in the range of 2-10 $\mathrm{nm}$, which meets the requirements of effective antimicrobial efficacy, (iv) the presence of Ag nanoparticles increases the porosity of the dressing facilitating the absorption of exudate from the wound and promoting the exchange of substances between cells, (v) the addition of Ag nanoparticles significantly improved the antibacterial ability of the material against $S$. aureus, (vi) ultimately constructed materials showed low cytotoxicity to mouse fibroblasts (L929 cells), which increased with the increase of Ag nanoparticles, (vii) the most biocompatible systems were that with the lowest silver content, however (viii) assessment of the wound healing effect on the rabbits showed that the system which contained $0.03 \% \mathrm{Ag}$ promoted skin regeneration and repair - after 10 days of infection, (ix) the wounds showed complete coverage of the new skin, creating a uniform, thick epithelial tissue and after 15 - the skin was covered with a uniform stratum corneum.

\section{CONCLUSIONS}

Tissue engineering is an interdisciplinary field that uses material engineering chemistry, biology, medicine and pharmacy to create natural substitutes that reproduce and improve tissue functions. It is generally accepted that this area of science is the future of regenerative medicine an attractive and effective alternative to such methods as transplants or prostheses, counteracting certain limitations resulting from their use, such as the risk of graft rejection or lack of integration with natural tissue.

Therefore, biomedical engineering is a dynamically developing field. This extraordinary progress is possible thanks to the continuous broadening of knowledge about biodegradable polymers. These materials can be successfully applied to create polymeric three-dimensional scaffolds designed to support the differentiation and proliferation of stem cells and, as a result, regeneration of damaged tissue. An undeniable advantage is their degradation in the body into non-toxic and easily excreted products, so that there is no need to remove implants from the patient's body, which significantly affects the comfort of his therapy. In addition, nanotechnology 
provides an incredible opportunity to fabricate intelligent tissue engineering scaffolds that offer a host of benefits. Successively modernized methods of their preparation and modification accelerate research which can end with obtaining functional organs for transplants, skin substitutes, or intelligent wound dressings. Therefore, it should be expected that the design and manufacture of new polymeric materials for biomedical applications will continue to be a very popular field of research.

\section{REFERENCES}

[1] R. Langer, J.P. Vacanti, Science, 260, 920-926, (1993).

[2] Q.L. Loh, C. Choong, Tissue Engineering: Part B, 19, 485-502, (2013).

[3] A. Pianco, F. Paladini, M. Pollini, Journal of Biomedical Materials Research Part B: Applied Biomaterials, 107, 7-18, (2019).

[4] B. Joseph, R. Augustine, N. Kalarikkal, S. Thomas, Materials Today Communications, 19, 319-335, (2019).

[5] S.M. Ahsan, M. Thomas, K.K. Reddy, S.G. Sooraparaju, A. Asthana, I. Bhatnagar, International Journal of Biological Macromolecules, 110, 97-109, (2018).

[6] M. Sheikholeslam, M. E. E. Wright, M. G. Jeschke, S. Amini-Nik, Advanced Healthcare Materials, 7, 1700897, (2018).

[7] C. Zhu, D. Fun, Z. Duan, X. Li, Y. Li, S. Wang, Y. Yu, Journal of Materials Science, 53, 5909-5928, (2018).

[8] C. Berce, M.S. Muresan, O. Soritau, B. Petrushev, L. Tefas, I. Rigo, G. Ungureanu, C. Catoi, A. Irimie, C. Tomuleasa, Colloids and Surfaces B, 163, 155-166, (2018).

[9] F. Croisier, G. Atanasova, Y. Poumay, C. Jérôme, Advanced Healthcare Materials, 3, 2032-2039, (2014).

[10] R. Singla, S.M.S. Abidi, A.I. Dar, A. Acharya, Journal of Biomedical Materials Research - Part B, (2019), DOI: 10.1002/jbm.b.34327.

[11] J. Liu, D.A. Sonshine, S. Shervani, R.H. Hurt. American Chemical Society Nano, 4, 6903-6913, (2010).

[12] A.F. Oyarzun, A. Vidal, M. Concha, J. Morales, S. Orellana, V.I. Moreno, Current Pharmaceutical Design, 21, 4329-4341, (2015).

[13] A. Wennerberg, V. Frojd, M. Olsson, U. Nannmark, L. Emanuelsson, P. Johansson, Y. Josefsson, I. Kangasniemi, T. Peltola, T. Tirri, Clinical Implant Dentistry and Related Research, 13, 184-196, (2011).

[14] A. Khalid, H. Ullah, M. Ul-Islam, R. Khan, S. Khan, F. Ahmad, T. Khan, RSC Advances, 7, 47662-47668, (2017). 
[15] N. Devi, J. Dutta, International Journal of Biological Macromolecules, 104, 1897-1904, (2017).

[16] D. Archana, B.K. Singh, J. Dutta, P.K. Dutta, Carbohydrate Polymers, 95, 530-539, (2013).

[17] T. Wang, Y. Zheng, Y. Shen, Y. Shi, F. Li, C. Su, L. Zhao, Artificial Cells, Nanomedicine, and Biotechnology, 46, 138-149, (2017).

[18] H. Ye, J. Cheng, K. Yu, International Journal of Biological Macromolecules, 121, 633-642, (2019). 